\title{
A Joint Model for Radio and $\gamma$-ray Emission from Pulsars
}

\author{
G. J. Qiao, K. J. Lee, H. G. Wang, and R. X. Xu \\ Astronomy Department, Peking University, Beijing 100871, P.R.China.
}

\begin{abstract}
Although pulsars can radiate electromagnetic wave from radio to gamma ray bands, we still have no a united model to understand the multi-band emission. In this paper the effort for a joint model is presented. The inverse Compton scattering (ICS) and a second acceleration process near the null surface are involved to account for the radio and the gama-ray emission, respectively. Various kind of pulse profiles and other observational properties can be reproduced.
\end{abstract}

\section{Introduction}

Pulsar is able to radiate multi-band emission. A wealth of observational data on radio pulsars has been collected since the discovery of pulsar. In high energy, 7 gamma-ray pulsars have been discovered by far. Gamma-ray photons from young pulsars allow the deepest insight into the properties of high-energy particles and their interaction with the magnetic fields and photons in pulsar magnetosphere. To understand the observational facts, polar cap models are proposed to account for the radio emission and both the polar cap and outer gap models are developed to explain the gamma-ray emission. In the past decades these two domains have been investigated separately. However, both radio and gamma-ray emissions can be radiated from the same pulsar, such as the Crab and the Vela pulsars, therefore it is highly necessary to establish a united model for radio and gammaray emissions.

\section{Radio emission from pulsars}

There are two kinds of polar cap models for pulsar radio emission. One is the inner gap model, which suggests that a gap-type accelerator could be formed above the polar cap surface (Ruderman \& Sutherland 1975), the other is spacecharge-limited flow model, which suggests that either the negative or the positive ions could flow freely from the stellar surface (Arons 1983). Although the binding energy of positive ions is not so high to form an inner gap on very hot neutron star surface, recent investigations found that inner gap may still exist in the situation of bare strange star (Xu et al. 2001) or in some cases of neutron star (Gil et al. 2002).

Any radio model is required to be able to explain the main observational facts, e.g., the pulse profile, the polarization, the spectrum and so on. We have proposed an inverse Compton scattering (ICS) model for radio emission based 


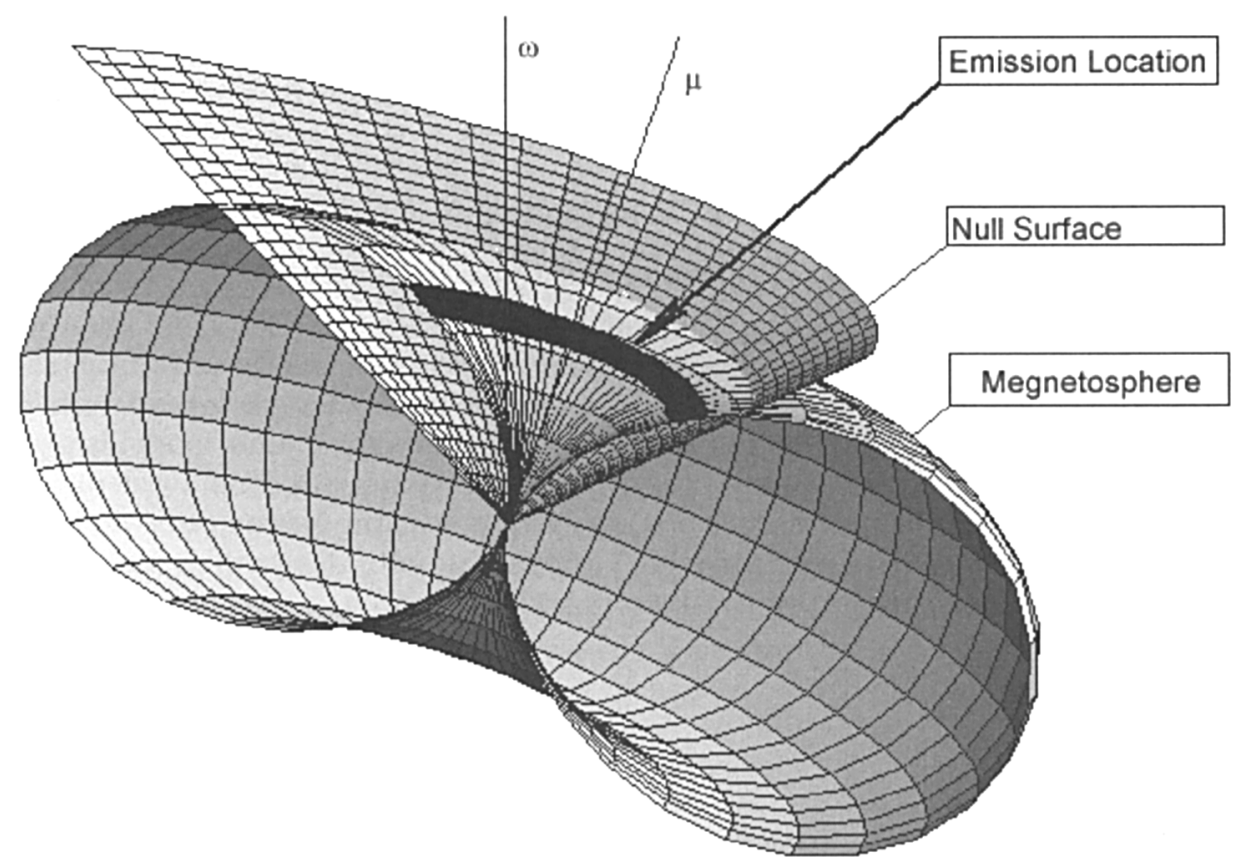

Figure 1. The null charge surface and the emission location.

on the inner gap scenario (see Qiao \& Lin 1998, hereafter paper I, Xu et al. 2000, Qiao et al. 2001). Under this model, some important observational properties can be reproduced: (1) the central (or "core") emission beam and the conal beams; (2) the location for each emission component; (3) the linear and circular polarization of individual and integrated pulses; (4) the pulse profiles changing with the frequency. The ICS model is involved in our joint model to account for the radio emission.

\section{Gamma-ray emission from pulsars}

Even if the local charge density $\rho$ of secondary particles just out of the inner gap is the same as that of local Goldreich-Julian density $\rho_{g j}\left(\rho_{g j}=-\boldsymbol{\Omega} \cdot \mathbf{B} /(2 \pi c)\right)$, as the particles stream out, the charge density should departure from the local $\rho_{g j}$; the acceleration caused by space-charge-limited flow will take place. The potential can be written as (Arons 1983)

$$
-\nabla^{2} \phi=4 \pi\left(\rho-\rho_{g j}\right)
$$

In the one-dimensional case, the electric field parallel to the magnetic field reads (Michel 1974)

$$
d E_{\|} / d z=4 \pi\left(\rho-\rho_{g j}\right)
$$




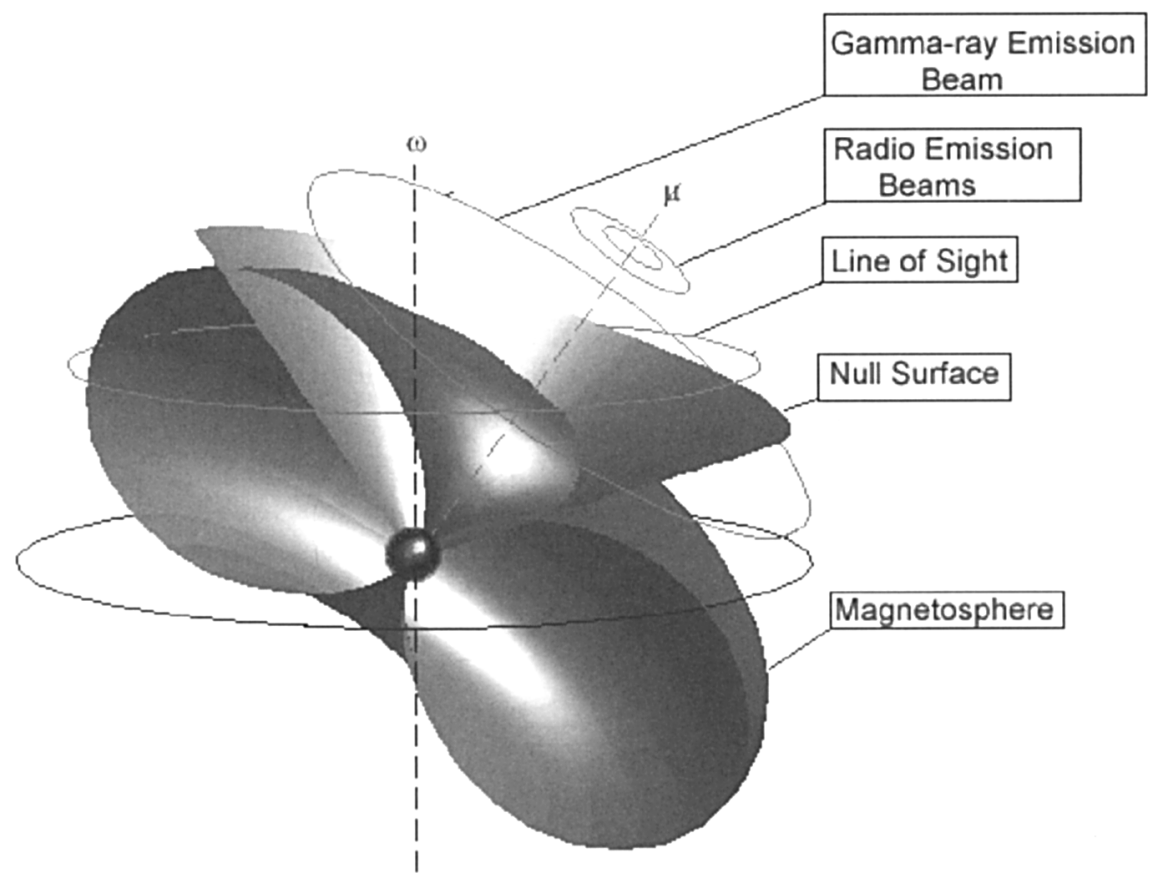

Figure 2. The pulsar's magnetosphere, null charge surface and emission beams. It is shown that the gamma-ray beam can be rather wide with the emission location inside the null charge surface.

In this way the particles will be accelerated effectively near the null surface where $\rho_{g j}=0$ and then radiate gamma-rays. The geometry of this acceleration location is shown in Fig.1 schematically. Such an acceleration process is effective within the bundle of open field lines that intersect the null surface.

The gamma-ray beam and light curve are figured out according to geometrical relations (Fig.2). Since the relative location of the secondary acceleration region to the polar cap surface depends on the inclination angle, the calculated gamma beam can be narrow or very wide, and the gamma and radio pulses may show various kinds of phase configuration, from alignment to a wide separation. For the comparison of calculated results with the observational profiles readers are referred to Qiao et al. (2002). The details of physical process are to be presented elsewhere.

\section{Conclusions and discussions}

From the geometrical consideration above one can see that both the inner and the outer gaps may play significant roles in pulsar radiation. The key point is that the inner gap acceleration and the space-charge-limited flow acceleration near the null surface should be taken into account. Low frequency waves supplied by the inner gap sparking are inverse Compton scattered by the outgoing relativistic 
particles to produce the radio emission (Paper I). The particles then encounter a secondary acceleration near the null charge surface and emit gamma-rays. Various kinds of observational properties can be reproduced. Although only a few pulsars were observed as gamma-ray pulsars, it is very possible that some pulsars can just not be observed in gamma-ray owing to our line of sight missing the emission beam. The joint model presented here is different from the current polar cap gamma-ray models because the null charge surface plays the vital role in secondary acceleration. Our model is also different from the present outer gap models: the secondary particles are accelerated by the effect of spacecharge-limited flow and the radiation location is extended inside into the null surface.

Acknowledgments. We are very grateful to Professor R.N. Manchester for his valuable discussion. This work is partly supported by NSF of China, and the Research Fund for the Doctoral Program Higher Education.

\section{References}

Arons, J., 1983, ApJ, 266, 215

Gil, J. \& Melikidze, G. I., 2002, ApJ, 577, 909

Michel, F. C., 1974, ApJ, 192, 713

Qiao, G. J. \& Lin W. P. 1998, A\&A, 333, 172 (PaperI)

Qiao, G. J., Liu, J. F., Zhang, B. \& Han, J. L. 2001, A\&A, 377, 964

Qiao, G. J., Lee, K. J., Wang, H. G. \& Xu, R. X., in "Radio Pulusars", ASP conference Sries, Vol. to be determined, 2003, eds., M. Bailes, D. J. Nice \& S. E. Thorsett

Ruderman, M. A. \& Sutherland, P. G., 1975, ApJ, 196, 51

Xu, R.X., Liu, J.F., Han, J.L., \& Qiao, G.J. 2000, ApJ, 535, 354

Xu, R.X., Zhang, B., \& Qiao, G.J. 2001, AstroParticle, 15, 101 